\title{
Implementación de un Laboratorio Virtual para la enseñanza de Controladores PID
}

\author{
David A. Márquez y Oscar O. Cárdenas \\ Universidad de Los Andes, Facultad de Ingeniería, Postgrado en Automatización e Instrumentación, \\ La Hechicera, Mérida-Venezuela (e-mail: damarquezg@ula.ve, oscaror@ula.ve)
}

\begin{abstract}
Resumen
El objetivo del trabajo es desarrollar un instrumento didáctico, que permita introducir variaciones de los parámetros del controlador para visualizar la influencia que tienen en la estabilidad de los procesos, con la finalidad de impartir enseñanza del control de procesos haciendo uso del computador. En los casos estudiados se fijan primero los valores de los parámetros y luego se deja libertad de insertarlos tanto en el controlador como en el proceso para analizar sus efectos. Las pruebas realizadas visualizan las ventajas y desventajas que el empleo de este tipo de controlador tiene para un proceso en particular. La experiencia obtenida con los estudiantes fue satisfactoria, logrando que los conceptos estudiados quedaran claros, en particular el tipo de variación a realizar en los parámetros del controlador para obtener mejor estabilidad de un sistema de control.
\end{abstract}

Palabras clave: controladores PID, enseñanza, simulación, laboratorio virtual

\section{Implementation of a Virtual Laboratory for teaching PID Controller}

\begin{abstract}
The objective of this work is to develop a teaching tool, which allows variations in the parameters of the controller to see the influence they have on the stability of this process, with the aim of teaching process control using the computer. In the cases studied the values of the parameters were first fixed and then the students are allowed to change them both in the controller and in the process, to analyze their effects. The tests conducted showed the advantages and disadvantages of the use of this type of controller in a particular process. The experience gained with the students was satisfactory and the concepts were well understood by the students, in particular the type of changes that need to be introduced to reach stability of the control system.
\end{abstract}

Keywords: PID controllers, teaching, simulation, virtual laboratory 


\section{INTRODUCCIÓN}

La educación a distancia se presenta como la solución idónea para un conjunto de colectivos que exigen el disponer de sistemas de enseñanza mucho más flexibles, accesibles y adaptables. Destacan dos herramientas como las más adecuadas para ofrecer un nuevo enfoque en el modelo educativo de la educación a distancia: los sistemas hipermedia como forma de estructurar la información, y las redes de comunicación de área extendida como soporte de la información, es decir, la red Internet (Antsaklis, 1999). La enseñanza de sistemas de control, requiere de un elemento adicional que permita al estudiante poner en práctica todos los conocimientos que vaya adquiriendo a lo largo del estudio de la materia. Este papel lo desempeña el laboratorio de prácticas, el cual, requiere de la presencia física del estudiante. Trasladando este entorno práctico a la enseñanza a distancia, el elemento necesario para la realización de prácticas es la existencia de un sistema de apoyo a la enseñanza consistente en un laboratorio virtual, que permita al alumno practicar de una forma lo más similar posible a como si estuviese en las dependencias del laboratorio. Para cumplir con este objetivo, en este trabajo se presentan dos aplicaciones las cuales pretenden facilitar el aprendizaje de los controladores PID. Como primera aplicación se presenta un tutorial basado en software donde se explica detalladamente y a través de simulaciones de diferentes procesos, cada estructura de control: P, PD, PI y PID. La segunda aplicación, presenta la implementación de este módulo de aprendizaje a través de Internet, lo que permitió desarrollar simulaciones basadas en la Word Wide Web (Sánchez et al., 2004). La finalidad última del proyecto va encaminada a crear las herramientas y el entorno adecuado para estructurar un laboratorio virtual, que permita una mejor formación práctica de nuestros estudiantes.

\section{METODOLOGIA}

La metodología utilizada para el diseño es la expuesta por Fenrich (2002), la cual contempla diez fases que fueron analizadas en su contexto particular para obtener las aplicaciones objeto de este trabajo. En las aplicaciones desarrolladas se muestra el contenido teórico en cuanto a controladores PID se refiere y se realizan simulaciones dependiendo del tipo de controlador que se quiera estudiar, teniendo la opción de manipular los valores del controlador en estudio (Bakosova et al., 2007). Cada aplicación fue estructurada de la siguiente manera tomado como base de partida las etapas que proponen Skogestad y Postlethwaite (1998), para este tipo de diseño: selección del proceso: primero o segundo orden; selección del controlador: P, PI, PD, PID; simulación con software de control dejando accesible la fijación de los parámetros del controlador y del proceso; simulación con valores preestablecidos y simulación con valores aleatorios para comparación. El uso de imágenes, textos y gráficas fue enfatizado para conseguir la motivación por el material. La navegación a través de las aplicaciones se realizó creando pantallas consistentes, de uso intuitivo, con menús controlados por simples aplicaciones de botones.

\section{RESULTADOS Y DISCUSION}

La pantalla del menú principal se muestra en la Figura 1a. Esta pantalla contiene básicamente los botones de acceso a cada una de las secciones en las que fue estructurada la aplicación: Introducción, Control P, Control PI, Control PD, Control PID, Ayuda y el botón Salir. A partir de la pantalla del menú principal, el usuario puede acceder a cada uno de los aspectos teóricos considerados relevantes para el entendimiento de los controladores PID. El usuario tiene acceso a toda la información organizada en forma de lecciones referente a cada tema, La Figura 1b, muestra la pantalla del submenú "Control P" la cual se despliega a partir del la pantalla del menú principal. Se puede observar cómo a la derecha de la ventana de texto hay unos botones etiquetados como " $>>$ " y "<<", los cuales sirven para avanzar o retroceder en la lección respectivamente, también hay un botón etiquetado como "Menú" el cual permite regresar a la pantalla de menú principal. Esta estructura ilustrada, se mantiene a lo largo de cada una de las lecciones, permitiendo al estudiante ir leyendo paso a paso cada uno de los componentes teóricos que conforman el tutorial. Para cada uno de los submenús correspondientes a cada tipo de control, se crearon simulaciones implementadas en Simulink que permiten complementar la parte teórica (Figura 1b). Al correr la simulación aparece una ventana correspondiente a la gráfica de la salida del sistema que se esta simulando. En la ventana de texto correspondiente a la parte teórica, se explica la finalidad de la simulación así como 
el procedimiento paso a paso de los parámetros a configurar para realizar la simulación. Una de las ventajas que presentan las simulaciones desarrolladas en la aplicación es que permiten la modificación de todos los parámetros de los bloques que conforman la simulación (Figura 1b). En esta figura se observa el caso del Controlador $\mathrm{P}$ que despliega una ventana correspondiente a su ganancia y en la cual se observa el valor por defecto que tiene en ese momento y dando la posibilidad de cambiar el parámetro para luego volver a simular y obtener así una nueva salida.
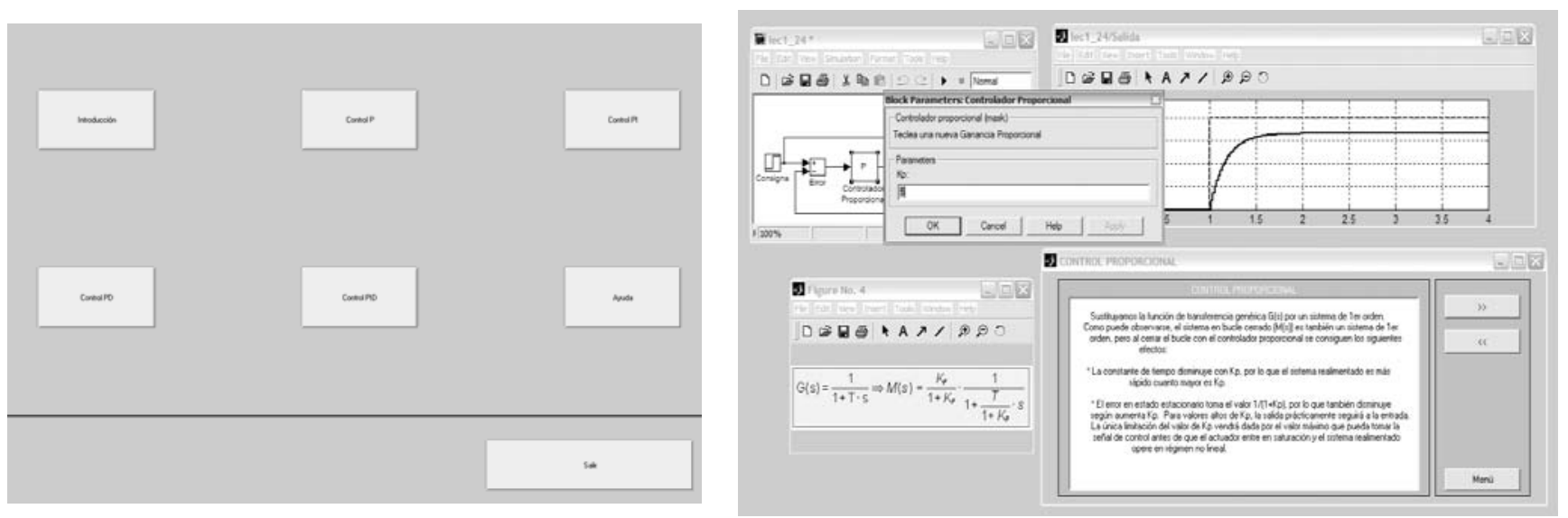

Fig. 1: (a) Pantalla del menú principal, (b) Pantalla del submenú "Control P"

Implementación Vía Internet - Simulación de Forma Remota

Basado en Internet se pueden encontrar dos opciones diferentes para el desarrollo de laboratorios: los laboratorios virtuales y los laboratorios remotos. Un laboratorio virtual permite, el acceso a procesos simulados en un computador. Los laboratorios remotos por su parte ofrecen experimentos reales a usuarios remotos (Aliane et al., 2007; Johansson, 1998). En base a estos conceptos, se desarrollo un laboratorio virtual en donde los estudiantes tienen acceso remoto a simulaciones y prácticas lo que permite profundizar el conocimiento adquirido en el aula de clase. Este desarrollo está basado en aplicaciones de Matlab, usando su herramienta Matlab Web Server (MWS), con el que se pueden realizar laboratorios virtuales de manera muy simple (Vallés, 2001). MWS es un software integrado que permite a los programadores de Matlab crear aplicaciones que utilizan las facilidades de la Word Wide Web para enviar datos a Matlab, mostrando posteriormente los resultados de los cálculos también vía web (Figura 2a). El proceso de desarrollo de aplicaciones MWS suele necesitar una combinación de modelos de Simulink, ficheros de Matlab y código HTML. En la Figura 2b, se presenta el esquema general de la aplicación. Utilizando el MWS como el corazón de la aplicación es posible desarrollar un laboratorio virtual que permita el acceso remoto a las simulaciones y procesos.
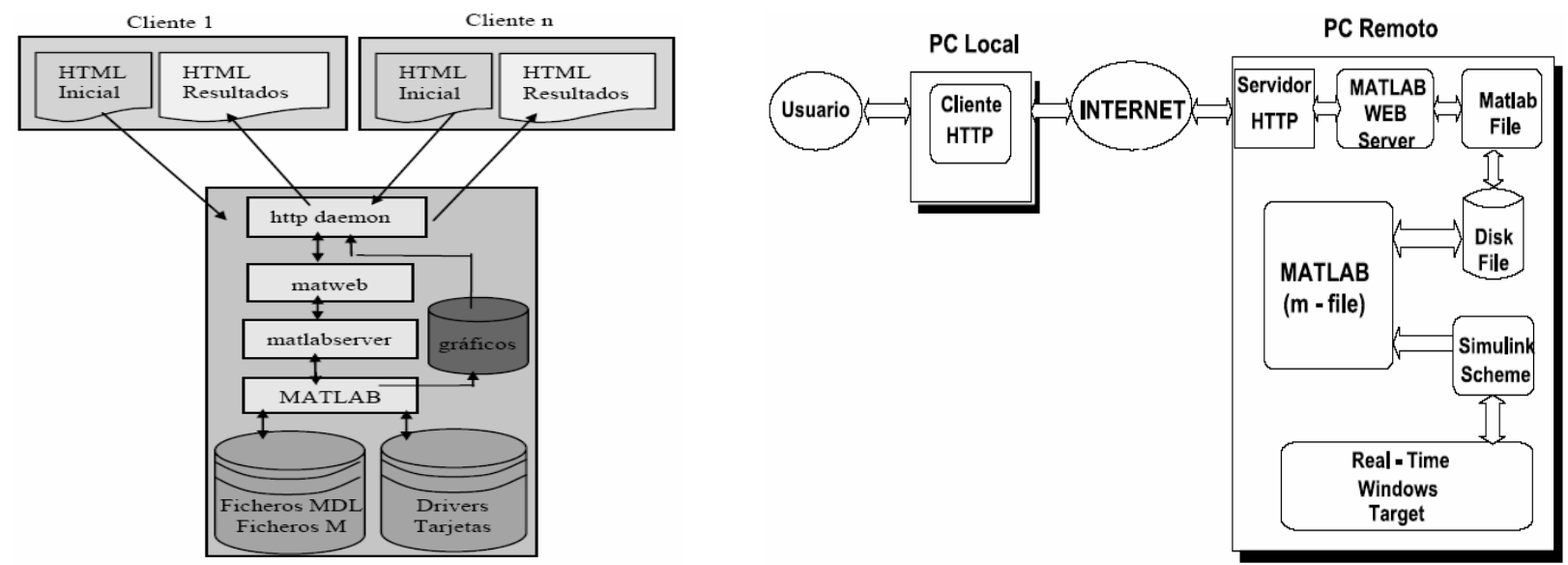

Fig. 2: (a) Estructura de ficheros de MWS, (b) Esquema general de la aplicación

El funcionamiento interno de la implementación se resume en la aplicación Web, la cual incluye la comunicación cliente - servidor utilizando los protocolos HTTP/HTML, el control de acceso de usuarios y la aplicación CGI (Common Gateway Interface) principal. La aplicación CGI se encarga del 
acceso a los recursos y la comunicación entre el servidor y las aplicaciones Matlab y Matlab Web Server. En la Figura 3 se muestra la aplicación desarrollada. En este caso el usuario sólo necesita un navegador de Web (Mozilla, Internet Explorer u otro) para acceder al sitio Web del laboratorio virtual.

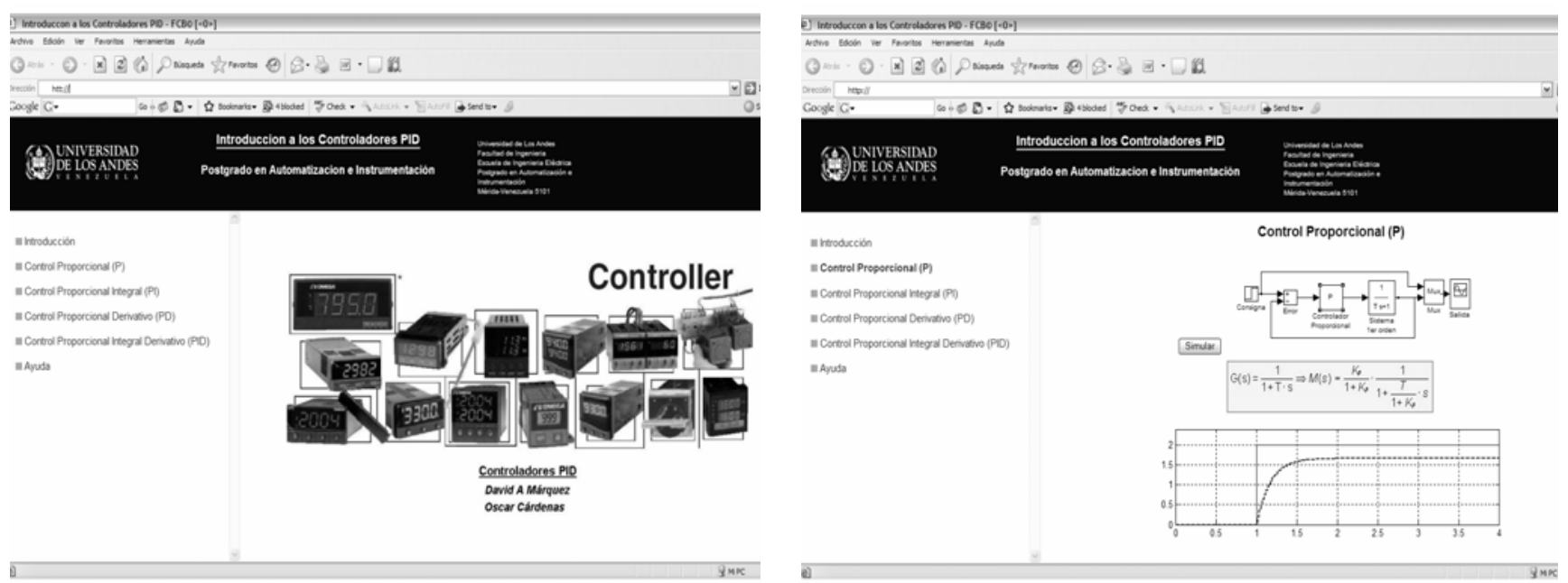

Fig. 3: Pagina Web del laboratorio (a) Página de inicio, (b) Pagina de simulación del Controlador P

\section{CONCLUSIONES}

La combinación de un entorno interactivo dotado de textos, imágenes y simulaciones promueve la participación activa de los estudiantes en su propio proceso de aprendizaje. La utilización de estas nuevas tecnologías ofrece una nueva posibilidad en la formación de los profesionales en las diferentes especialidades de la ingeniería. En este trabajo se presentaron dos herramientas para el aprendizaje de Controladores PID, que no pretende sustituir la labor del docente, sólo le sirve de apoyo en las clases, para elevar la calidad del proceso de aprendizaje. Desde el punto de vista de control, las herramientas desarrolladas permite al alumno familiarizarse con tareas y conceptos tales como la supervisión del proceso, cambios de los modelos de la planta, cambios en los algoritmos de control, cambios en los parámetros del controlador, entre otros.

\section{REFERENCIAS}

Aliane N., J. Fernández, A. Martínez y J. Ortiz; "Un Laboratorio de Ingeniería de Control Basado en Internet” Inf. Tecnológica: ISSN: $0718-0764$ (en línea), 18(6), 19-26. (2007), http://www.scielo.cl/pdf/ infotec/v18n6/art04.pdf. Acceso: 10 Enero (2008)

Antsaklis, P., T. Basar, R. DeCarlo y N. Harris; "Report on the NSF/CSS WorkShop on New Directions in Control Engineering Education", IEEE Control Systems Magazine, 19(5), 53-58 (1999).

Bakosova M., M. Fikar, L. Cirka. "E-learning in Process Control Education", European Congress of Chemical Engineering (ECCE-6), Copenhagen 16-21 September (2007).

Fenrich F.; Practical guidelines for creating instructional multimedia applications, The Dryden Press, USA (2002).

Johansson, M.; M. Gäfvert y K.J. Åström; "Interactive tools for education in automatic control”, IEEE Control Systems Magazine, 18(3), 33-40 (1998).

Sánchez, J., F. Morilla y S. Dormido; "An environment of remote visual supervision over the Internet for educational purposes", IEEE Trans on Education: 42(3), 180-185 (2004).

Skogestad, S. y Y. Postlethwaite; "Multivariable feedback control”, IEEE Control Systems Magazine, 18(3), 26-32 (1998).

Vallés, M., J.L. Díez y A Valera; "Setting up a Virtual Matlab Control Laboratory", IFAC-Workshop EDCOM2001: Education in Automatic Control. Hohannes Kepler University linz, Austria (2001). 